\title{
Compatibilidad entre pensiones contributivas por incapacidad y empleo: el caso español ${ }^{1}$
}

\author{
Miguel Ángel MALO \\ Universidad de Salamanca \\ malo@usal.es \\ Begoña CUETO \\ Universidad de Oviedo \\ bcueto@uniovi.es \\ Vanesa RODRÍGUEZ \\ Universidad de Oviedo \\ rodriguezvanesa@uniovi.es
}

Recibido: 7-4-10

Aceptado: 26-5-10

\section{RESUMEN:}

En este artículo analizamos la compatibilización de las pensiones contributivas por incapacidad permanente con el trabajo remunerado. Mediante la Muestra Continua de Vidas Laborales se ha obtenido que un 9,9\% de los trabajadores se encuentra en 2006 en esta situación, alcanzado un 15,6\% en el caso de la incapacidad permanente total. El principal determinante de la compatibilización es cobrar una pensión por incapacidad permanente total o absoluta, es decir, la gravedad de la discapacidad sufrida. Una vez tenido en cuenta el efecto del tipo de pensión, la cuantía de la misma no desincentiva la compatibilización.

Palabras clave: incapacidad permanente, discapacidad, pensión por incapacidad.

Compatibility between incapacity pensions and employment: the Spanish case

\begin{abstract}
:
In this article, we analyze the compatibility between contributory incapacity pensions and employment. Using the Spanish Working Lives Longitudinal Database, we show that $9.9 \%$ of workers are in this situation, reaching $15.6 \%$ for total permanent incapacity. The main determinant of this simultaneity is the severity of the
\end{abstract}

${ }^{1}$ Este trabajo se presentó en el encuentro Economic Well-Being and Social Protection in Southern Europe: Changing Policies and Trends organizado por el Instituto de Estudios Fiscales el 15 y 16 de noviembre de 2007 en Madrid. Los autores agradecen a los asistentes sus comentarios y sugerencias, en especial a José María Labeaga y a José María Arranz. Para el desarrollo de esta investigación se ha obtenido financiación del Fondo de Investigación sobre la Protección Social (FIPROS). Referencia MTIN-FIPROS-2008/39. Dicha institución no es responsable de las opiniones de los autores ni tiene por qué asumir los resultados de la presente investigación. 
disability (using the type of pension as a proxy). Once we consider the type of pensions, we do not find a relevant effect of the amount of the pension on such compatibility.

Key words: permanent incapacity, disability, incapacity pensions.

\section{REFERENCIA NORMALIZADA}

Malo, M.A., Cueto, B. y Rodríguez, V. (2011). Compatibilidad entre pensiones contributivas por incapacidad y empleo: el caso español”. Cuadernos de Relaciones Laborales Vol. 29 Num. 1, p. 125-153.

SUMARIO: 1. Introducción. 2. Prestaciones por incapacidad permanente e incentivos al trabajo. 2.1. Grados de incapacidad permanente. 2.2. Requisitos de acceso y cuantía de la prestación por incapacidad. 3. Análisis empírico. 3.1. Datos y definiciones de las principales variables. 3.2. Análisis descriptivo. 3.3. Análisis econométrico. 3.4. La reforma de diciembre de 2007: una simulación. 4. Conclusiones. 5. Bibliografía. Apéndice.

\section{Introducción}

La cobertura de las situaciones tradicionalmente llamadas de "invalidez" o "incapacidad" permanentes (denominaciones que siguen perviviendo en buena parte del lenguaje administrativo aunque hoy en día hayan sido sustituidas entre los especialistas por el término discapacidad) es sin duda uno de los logros históricos del Estado de Bienestar. Esa cobertura se entendió siempre como una garantía de ingresos regulares que permitieran a estos individuos el no caer en situaciones de extrema necesidad o no quedar al amparo exclusivo de sus familias. No obstante, en las últimas décadas se ha ido desarrollando una nueva perspectiva según la cual no basta con el diseño de un sistema de transferencias públicas, sino que para la plena integración social de las personas con discapacidad el acceso al empleo se ve como un elemento esencial.

En este contexto de cambio en la concepción de las políticas hacia las situaciones de discapacidad ha comenzado a desarrollarse un debate sobre qué tipo de encaje deben mantener los sistemas de transferencias (contributivas y no contributivas) relacionadas con la discapacidad y la promoción del empleo entre las personas con discapacidad. Tradicionalmente, la investigación internacional (Parsons, 1980; Bound y Burkhauser, 1999; Bound et al., 1999) se ha centrado en analizar el papel de las pensiones por discapacidad como desincentivo para trabajar y convertirse en una vía indirecta para la jubilación anticipada. En cuanto al caso español, los análisis existentes están más bien centrados en el estudio de la jubilación anticipada $\mathrm{y}$, de forma complementaria, tratan el eventual uso de las pensiones por discapacidad por parte de los trabajadores mayores para salir definitivamente del mercado de trabajo, como es el caso de Blanco (2000), Jiménez (2006), Díaz-Jiménez y Díaz-Saavedra (2006). Más allá del caso de los trabajadores mayores, Malo (2004) al analizar la participación laboral de las personas con discapacidad en edad laboral (y no sólo las próximas a la jubilación por vejez) encuentra que recibir cualquier tipo de prestación o subsidio tiene un efecto negativo sobre la participación en el mercado de trabajo de este colectivo, mostrando que probablemente el factor más importante detrás de este efecto negativo no es la tasa de sustitución sino el diseño de las transferencias de ingresos. 
Nuestro trabajo se sitúa en esa línea, pero nuestro interés se dirige hacia todo el colectivo de personas con discapacidad en edad laboral y no meramente al estudio del eventual uso estratégico del sistema de pensiones por discapacidad por parte de los trabajadores mayores. Nuestro objetivo consiste en conocer los determinantes de la compatibilización entre pensiones por incapacidad y empleo en el caso de España. La relevancia política y social de incrementar el conocimiento sobre este tema tiene que ver con que los individuos puedan usar la "capacidad parcial" que puedan tener y tener así acceso a un empleo, con lo que esto conlleva de normalización y plena participación social de las personas con discapacidades. Una de las variables a la que prestaremos especial atención es a la cuantía de la pensión y su influencia sobre la probabilidad de compatibilización.

Como objetivo secundario, también realizamos una simulación de los eventuales efectos sobre la compatibilidad entre pensión por incapacidad y empleo de los cambios legales introducidos en el diseño de estas pensiones mediante la Ley 40/2007 de Medidas en materia de Seguridad Social. Aunque dichos cambios no tenían como objetivo fomentar dicha compatibilización, alteran condiciones que, como veremos, afectan a variables que influyen en la probabilidad de que un individuo con pensión por incapacidad la compatibilice con un empleo.

\section{Prestaciones por incapacidad permanente e incentivos al trabajo}

La incapacidad permanente se define como una situación en la que el trabajador, después de haber estado sometido al tratamiento prescrito y de haber sido dado de alta médicamente, presenta reducciones anatómicas o funcionales graves, susceptibles de determinación objetiva y previsiblemente definitivas, que disminuyen o anulan su capacidad laboral.

\subsection{Grados de incapacidad permanente}

Existen cuatro grados de incapacidad permanente (IP) que conllevan su correspondiente prestación: incapacidad permanente parcial para la profesión habitual; incapacidad permanente total para la profesión habitual; incapacidad permanente absoluta para todo trabajo; y gran invalidez ${ }^{2}$.

La incapacidad permanente parcial (IPP) para la profesión habitual es aquella que ocasiona al trabajador una disminución no inferior al 33\% en su rendimiento normal para la profesión habitual, sin impedirle la realización de las tareas fundamentales de

${ }^{2}$ La Ley 24/1997 modificó el artículo 137 LGSS donde se establecen los distintos tipos de incapacidad permanente, sin embargo también incluyó una disposición transitoria que indicaba que la nueva redacción sólo sería de aplicación una vez que entrara en vigor el desarrollo reglamentario de dicho artículo. Hasta la fecha no se ha llevado a cabo dicho desarrollo reglamentario. 
la misma. Esta situación es compatible con el desarrollo de cualquier actividad laboral, tanto por cuenta ajena como por cuenta propia y con el mantenimiento del trabajo que se viniera desarrollando.

La incapacidad permanente total (IPT) para la profesión habitual es una situación que inhabilita al trabajador para la realización de todas o de las fundamentales tareas de dicha profesión, siempre que pueda dedicarse a otra distinta. Esta prestación es incompatible con el desempeño del mismo puesto en la empresa, pero compatible con la realización de cualquier trabajo por cuenta ajena o propia en la misma empresa o en otra distinta, si bien el pensionista debe comunicar a la Seguridad Social dicha circunstancia.

La incapacidad permanente absoluta (IPA) para todo trabajo supone que el trabajador está inhabilitado por completo para toda profesión u oficio. La pensión no impide el ejercicio de aquellas actividades, sean o no lucrativas, compatibles con el estado del inválido y que no representen un cambio en su capacidad de trabajo a efectos de revisión. Si se realizan trabajos susceptibles de inclusión en alguno de los regímenes de la Seguridad Social, existe la obligación de cursar la correspondiente alta y cotizar, debiendo comunicar el pensionista a la Entidad gestora el inicio de la actividad, ya sea por cuenta ajena o propia.

Finalmente, en el caso de gran invalidez (GI), el trabajador, a consecuencia de pérdidas anatómicas o funcionales, necesita la asistencia de otra persona para los actos más esenciales de la vida, tales como vestirse, desplazarse, comer o análogos. La compatibilidad con el trabajo es análoga a la de la incapacidad permanente absoluta.

En cuanto a cómo se compatibilización estas pensiones con el empleo, la pensión por IPT está concebida precisamente para ello. Sin embargo, también es posible la compatibilización de las pensiones por IPA y GI con un empleo. Cuando se reconoce una pensión por IPA o GI, la normativa nos dice que se está dando esa prestación económica por no poder desarrollar ninguna profesión, por lo que sólo se podrían desarrollar tareas laborales que no sean el núcleo funcional de ninguna profesión u oficio $^{3}$. Sin embargo, las dificultades para determinar qué tareas configuran ese núcleo funcional y cuáles no genera una incertidumbre importante, a lo que se añade que los cambios tecnológicos pueden alterar de manera relevante qué tareas puede realizar una persona (piénsese, por ejemplo, en las aplicaciones de la informática y la electrónica a las ayudas técnicas disponibles para las personas con discapacidades relacionadas con el movimiento). En cualquier caso, lo que cabe esperar es que, por definición,

${ }^{3}$ De hecho, una vez que una persona con este tipo de pensión comunica que está trabajando, la Seguridad Social suele iniciar un proceso para rebajar la calificación de la pensión al menos a la de incapacidad permanente total (si las tareas que se ejercen son de una familia profesional distinta de la original del individuo). Los beneficiarios suelen recurrir ante los tribunales la decisión de la Seguridad Social. En esos recursos, parece ser que la jurisprudencia está limitando esas decisiones de "rebaja" a categorías inferiores de pensión contributiva por incapacidad (Blanco, 2002). 
la compatibilización se produzca más frecuentemente en el caso de la IPT y que sea más bien ocasional entre los casos de IPA y GI.

\subsection{Requisitos de acceso y cuantía de la prestación por incapacidad}

Los requisitos que deben cumplir las personas que quieran acceder a una prestación por incapacidad permanente se han mantenido estables durante el periodo 1985$2007^{4}$. Dado que los datos utilizados en el análisis empírico se refieren al año 2006, explicaremos con detalle los requisitos y cuantías vigentes en dicho año y nos referiremos a la reforma habida en 2007 en un apartado posterior para valorar las posibles consecuencias de los cambios establecidos en dicho año.

Desde 1985 hasta 2007, para ser beneficiario de las prestaciones económicas por incapacidad permanente hay que cumplir los siguientes requisitos ${ }^{5}$ :

1. Tener menos de 65 años en la fecha en que comienza la situación de incapacidad y/o, si la incapacidad deriva de contingencias comunes, no reunir los requisitos exigidos para acceder a la pensión de jubilación contributiva del Sistema (quince años de cotización).

2. Estar afiliado y en alta o situación asimilada a la de alta, aunque en el caso de IPA y GI este requisito no es necesario.

3. Los requisitos relativos a la cotización cambian en función del grado de la incapacidad y de la causa de la misma tal y como se muestran en el Cuadro 1. En caso de riesgos profesionales o accidente laboral no existe ningún requisito mientras que si se trata de enfermedad común los requisitos varían en función de la edad y la situación de alta ${ }^{6}$.

La cuantía y la base reguladora de la prestación también difieren en función del grado de la incapacidad y de la causa de la misma. Respecto a la cuantía, se trata de una cantidad fija en el caso de la incapacidad permanente parcial, igual a 24 mensualidades de la base reguladora que sirve para calcular la incapacidad temporal (IT). En los restantes tipos de incapacidad permanente se calcula como un porcentaje de la base reguladora que es del $55 \%$ para la IPT y del $100 \%$ para la IPA y la GI.

Para la IPT existe la alternativa de una indemnización a tanto alzado de entre 12 y 84 mensualidades si el trabajador es menor de 60 años. Asimismo, el porcentaje se incrementa a partir de los 55 años en un $20 \%$ siempre y cuando la persona no trabaje.

\footnotetext{
${ }^{4}$ Para una revisión de la situación legal previa y de la reforma llevada a cabo en 1985 puede consultarse Álvarez (1982) y Desdentado et al. (1986) respectivamente.

${ }^{5}$ Tanto los requisitos como la cuantía de la prestación se refieren a la normativa vigente en noviembre de 2007 (Real Decreto Legislativo 1/1994).

${ }^{6}$ Una cuestión que no trataremos aquí es la de la valoración médica de la incapacidad laboral y su relación con los conceptos legales. Para los interesados en el tema de la valoración médica de la incapacidad laboral, Borobia (2007) es una fuente extremadamente detallada. Sobre los posibles cambios en la exigencia de los criterios en los noventa respecto de los ochenta véase Jiménez (2006), y para una crítica de esa posición López Arriba y Calvo Fernández (1989).
} 
En el caso de GI, se incrementa en un $50 \%$ o existe la posibilidad de ingresar en una institución a cargo del Estado. Como se ha mencionado, la base reguladora cambia en función de la causa de la incapacidad permanente y de su grado. En el Cuadro 1 se muestran las formas de cálculo en las distintas posibilidades.

\section{Cuadro 1. Requisitos de cotización previa para el acceso a prestaciones por incapacidad permanente y base reguladora}

\begin{tabular}{|c|c|c|c|c|}
\hline Causa de la IP: & $\begin{array}{l}\text { Incapacidad } \\
\text { permanente } \\
\text { parcial }\end{array}$ & $\begin{array}{l}\text { Incapacidad } \\
\text { permanente } \\
\text { total }\end{array}$ & $\begin{array}{l}\text { Incapacidad } \\
\text { permanente } \\
\text { absoluta }\end{array}$ & $\begin{array}{c}\text { Gran } \\
\text { invalidez }\end{array}$ \\
\hline \multicolumn{5}{|c|}{ Requisitos de cotización previa para el acceso a prestaciones por IP } \\
\hline $\begin{array}{l}\text { Riesgos profe- } \\
\text { sionales o } \\
\text { accidente no } \\
\text { laboral }\end{array}$ & \multicolumn{4}{|c|}{ Ninguno } \\
\hline \multicolumn{5}{|l|}{$\begin{array}{l}\text { Enfermedad } \\
\text { común: }\end{array}$} \\
\hline $\begin{array}{l}\text { + situación de } \\
\text { alta o asimila- } \\
\text { da y }>26 \text { años }\end{array}$ & \multirow{2}{*}{$\begin{array}{l}1.800 \text { días en los } \\
\text { últimos } 10 \text { años (si } \\
<21 \text { años: } 1 / 2 \text { del } \\
\text { tiempo trascurrido } \\
\text { entre los } 16 \text { años y } \\
\text { la IT más } 18 \\
\text { meses) }\end{array}$} & \multicolumn{3}{|c|}{$\begin{array}{c}\text { 1/4 del tiempo trascurrido entre los } 20 \text { años y la fecha } \\
\text { de la IP (mínimo } 5 \text { años) ( } 1 / 5 \text { parte en los últimos } 10 \\
\text { años) }\end{array}$} \\
\hline $\begin{array}{l}+ \text { situación de } \\
\text { alta o asimila- } \\
\text { da } y<26 \text { años }\end{array}$ & & \multicolumn{3}{|c|}{$\begin{array}{l}\text { La mitad del tiempo trascurrido entre los } 16 \text { años y la } \\
\text { fecha de la IP }\end{array}$} \\
\hline $\begin{array}{l}+ \text { situación de } \\
\text { no alta }\end{array}$ & - & - & \multicolumn{2}{|c|}{$\begin{array}{l}\text { Mínimo de } 15 \text { años ( } 3 \text { en los } \\
\text { últimos } 10 \text { años) }\end{array}$} \\
\hline
\end{tabular}




\begin{tabular}{|c|c|c|}
\hline \multicolumn{3}{|c|}{ Base Reguladora } \\
\hline \multirow[t]{2}{*}{$\begin{array}{l}\text { Enfermedad } \\
\text { común: }\end{array}$} & \multirow{4}{*}{$\begin{array}{l}\text { Base de cotización en } \\
\text { el mes anterior al } \\
\text { inicio de la IT / } \mathrm{n}^{\circ} \\
\text { días cotizados }\end{array}$} & $\begin{array}{l}B R=\frac{\sum_{i=1}^{24} B i+\sum_{i=25}^{96} B i * \frac{I_{25}}{I_{1}}}{112} \\
\mathrm{~B}_{\mathrm{i}}: \text { Base de cotización del mes i-ésimo anterior al } \\
\text { hecho causante; } \mathrm{I}_{\mathrm{i}}: \text { IPC del mes i-ésimo anterior al } \\
\text { hecho causante }(\mathrm{i}=1,2, \ldots, 96) \text {. Excepciones: a) si } \\
<52 \text { años: } \mathrm{BR}=\Sigma \mathrm{B}_{\mathrm{i}} /\left(\mathrm{n}^{\circ} \text { meses } * 1,1666\right) ;\end{array}$ \\
\hline & & $\begin{array}{c}\text { b) si situación de no alta, la fórmu- } \\
\text { la se aplica a los } 96 \text { meses inme- } \\
\text { diatamente anteriores al mes en } \\
\text { que se produce la IP }\end{array}$ \\
\hline \multirow[t]{2}{*}{$\begin{array}{l}\text { Accidente no } \\
\text { laboral: }\end{array}$} & & $\begin{array}{c}\mathrm{BR}=\Sigma \mathrm{B}_{\mathrm{i}} \text { durante un periodo ininterrumpido de } 24 \\
\text { meses (a escoger entre los } 7 \text { últimos años) / } 28 \\
\text { (si no se hubieran completado } 24 \text { meses, otra } \\
\text { opción: } \\
\Sigma \text { bases mínimas en los } 24 \text { meses anteriores a la } \\
\text { IP / } 28)\end{array}$ \\
\hline & & $\begin{array}{l}\text { b) si situación de no alta, se } \\
\text { calcula igual que en el caso de } \\
\text { enfermedad común }\end{array}$ \\
\hline $\begin{array}{l}\text { Contingencias } \\
\text { profesionales }\end{array}$ & $\begin{array}{l}\text { (Base de cotización } \\
\text { por cotizaciones } \\
\text { profesionales del mes } \\
\text { anterior a la baja } \\
\text { médica / } \mathrm{n}^{\circ} \text { días } \\
\text { cotizados) + prome- } \\
\text { dio de cotización por } \\
\text { horas extra en el año } \\
\text { anterior }\end{array}$ & $\begin{array}{c}\mathrm{BR}=(1 / 12) * \sum \text { (sueldo y antigüedad diarios en la } \\
\text { fecha de la IT } * 365)+(\text { pagas extra, beneficios o } \\
\text { participaciones en el año anterior })+([(\text { pluses, } \\
\text { retribuciones complementarias }+ \text { horas extra del } \\
\left.\left.\text { año anterior }) / \mathrm{n}^{\circ} \text { días trabajados }\right]^{* 273}\right)\end{array}$ \\
\hline
\end{tabular}

NOTA: BR representa la Base Reguladora.

\section{Análisis empírico}

\subsection{Datos y definición de las principales variables}

La Muestra Continua de Vidas Laborales de 2006 es la base de datos utilizada en esta investigación e incluye todas aquellas personas que han tenido algún tipo de relación laboral con la Seguridad Social en 2006 (trabajo, prestación contributiva por desempleo o pensiones contributivas) y, para cada una de ellas, toda su trayectoria laboral desde su primer empleo. Su diseño es retrospectivo, pues proporciona una imagen representativa de la población que mantiene alguna relación con la Seguridad Social en 2006, pero no necesariamente en los momentos del pasado para los cuales 
suministra información. Nos centraremos en las personas que en 2006 reciben una prestación por incapacidad permanente y, simultáneamente, tienen un empleo.

Para nuestro análisis, hemos seleccionado a las personas menores de 65 años y que reciben una prestación por incapacidad permanente en 2006. Para cada una de ellas hemos reconstruido su trayectoria laboral, atendiendo sobre todo al momento en el que se reconoce la IP. No se han considerado las prestaciones por incapacidad permanente parcial puesto que se trata de indemnizaciones a tanto alzado y no un ingreso mensual regular.

Nuestra selección recoge datos de 27.807 personas, de las cuales un 9,9\% están trabajando en 2006 al tiempo que reciben una prestación. Este porcentaje es más elevado si se considera el periodo de tiempo trascurrido desde el reconocimiento de la incapacidad permanente, ascendiendo a un $22,2 \%$, lo cual muestra que haber compatibilizado la pensión por incapacidad y el empleo no es una situación mayoritaria, pero es algo que dista de ser anecdótico.

El porcentaje más alto se da en el caso de la IPT, como se podía esperar puesto que se trata del grupo que, explícitamente según la normativa, puede realizar un trabajo distinto al que era habitual. Las cifras son sustancialmente más bajas tanto para la IPA como para la GI (véase Cuadro 2).

Para llevar a cabo el objetivo propuesto la población de referencia está formada por las personas que reciben una prestación que no sea una pensión de jubilación y que tengan menos de 65 años, para centrarnos en las personas en edad laboral. Los datos que se muestran en los cuadros que siguen se refieren a este grupo. Además, las variables relativas a su trayectoria laboral corresponden a ésta hasta el momento en que reciben la prestación, es decir, se trata de la trayectoria laboral antes de tener derecho a la pensión ${ }^{7}$.

\subsection{Análisis descriptivo}

Las características de las personas que reciben una prestación en función de si trabajan al mismo tiempo o no se muestran en el Cuadro 2. Entre las personas que trabajan hay más hombres y son relativamente más jóvenes que el grupo de personas que reciben prestación y no trabajan. Además, también han tenido su primer empleo más jóvenes y el reconocimiento de la pensión ha sido cuando tenían menos edad (menos de 40 años frente a 47). Asimismo, han trabajado menos tiempo y la duración media de sus episodios de empleo ha sido menor. Por otra parte, se puede indicar que un porcentaje pequeño, tanto del grupo de trabajadores como del de no trabajadores, reciben pensiones no ligadas al trabajo (de viudedad $\mathrm{u}$ orfandad).

\footnotetext{
${ }^{7}$ En el Cuadro A.1 del anexo se describen las principales variables utilizadas en el análisis.
} 


\section{Cuadro 2. Características de las personas que reciben prestación por incapaci-} dad permanente según la situación en alta y sexo

\begin{tabular}{|c|c|c|c|c|c|c|c|}
\hline & \multicolumn{3}{|c|}{ No trabaja en 2006} & \multicolumn{3}{|c|}{ Sí trabaja en 2006} \\
\hline & & Total & Mujer & Hombre & Total & Mujer & Hombre \\
\hline \multicolumn{2}{|c|}{ Sexo (\% hombres) } & $67,0 \%$ & & & $87,7 \%$ & & \\
\hline \multicolumn{2}{|l|}{ Edad: } & & & & & & \\
\hline \multicolumn{2}{|c|}{ En 2006} & 53,8 & 53,4 & 54,0 & 47,5 & 47,1 & 47,5 \\
\hline \multicolumn{2}{|c|}{ En el primer empleo } & 24,6 & 27,1 & 23,3 & 20,4 & 21,8 & 20,2 \\
\hline \multicolumn{2}{|c|}{ En el inicio de la IP } & 46,7 & 46,9 & 46,5 & 38,0 & 39,3 & 37,8 \\
\hline \multicolumn{2}{|c|}{ Discapacidad } & $1,2 \%$ & $1,3 \%$ & $1,2 \%$ & $1,7 \%$ & $2,3 \%$ & $1,6 \%$ \\
\hline \multicolumn{2}{|c|}{ Tiempo trabajado (años) } & & & & & & \\
\hline \multicolumn{2}{|c|}{$\begin{array}{l}\text { Tiempo potencial de trabajo (has- } \\
\text { ta la IP)* }\end{array}$} & 22,0 & 19,8 & 23,1 & 17,6 & 17,5 & 17,6 \\
\hline \multicolumn{2}{|c|}{ Tiempo total trabajado } & 17,3 & 14,6 & 18,6 & 14,1 & 12,5 & 14,3 \\
\hline \multicolumn{2}{|c|}{$\begin{array}{l}\text { Tiempo trabajado /tiempo poten- } \\
\text { cial }(\%)\end{array}$} & $78,5 \%$ & $73,7 \%$ & $80,5 \%$ & $79,9 \%$ & $71,4 \%$ & $81,1 \%$ \\
\hline \multicolumn{2}{|c|}{ Número de episodios de empleo } & 10,8 & 8,5 & 11,9 & 10,8 & 8,5 & 11,1 \\
\hline \multicolumn{2}{|c|}{$\begin{array}{l}\text { Duración media de los empleos } \\
\text { (años) }\end{array}$} & 4,4 & 4,8 & 4,2 & 2,9 & 3,3 & 2,9 \\
\hline \multicolumn{2}{|c|}{$\begin{array}{l}\text { Recibe prestación por viude- } \\
\text { dad/orfandad }\end{array}$} & $2,9 \%$ & $7,3 \%$ & $0,7 \%$ & $1,3 \%$ & $6,2 \%$ & $0,6 \%$ \\
\hline \multicolumn{8}{|c|}{ Cuantía de la pensión (media anual, $€$ ): } \\
\hline \multirow[b]{2}{*}{ IPT } & Trabaja en 2006 (\%) & & & & & & \\
\hline & 15,6 & $7.994,07$ & $6.351,74$ & $8.909,30$ & $\begin{array}{l}7.043,93 \\
10.291,5\end{array}$ & $5.959,33$ & $7.188,95$ \\
\hline IPA & 1,2 & $12.829,89$ & $11.595,71$ & $13.368,24$ & & $9.967,60$ & $10.379,60$ \\
\hline GI & 1,6 & $19.891,05$ & $17.671,38$ & $20.532,04$ & $\begin{array}{r}15.981,6 \\
4\end{array}$ & $17.701,42$ & $15.452,47$ \\
\hline
\end{tabular}

* El tiempo potencial de trabajo se refiere al tiempo trascurrido entre la fecha de inicio del primer contrato y la fecha de reconocimiento de la IP.

Fuente: Cálculos de los autores a partir de la MCVL-2006.

Si se tienen en cuenta las diferencias por sexo, se pueden señalar algunos rasgos presentes habitualmente en el mercado de trabajo. Las mujeres empiezan a trabajar más tarde y tienen menos experiencia laboral. No obstante, las diferencias son mayores cuando se considera si las personas que reciben una prestación tienen trabajo o no que si se presta atención a las diferencias entre sexos. Es decir, de cara al objeto de estudio es más relevante tener o no trabajo que ser hombre o mujer. 
Como ya se ha explicado, las diferencias en el cálculo de la cuantía de la prestación determinan que ésta sea mayor en el caso de gran invalidez y menor para la incapacidad permanente total, lo que se puede apreciar en el

Gráfico 1, en el que se muestra la distribución de la cuantía (en euros/año) según el grado de la pensión. Mientras que para la IPA y la IPT el porcentaje se acumula en los tramos inferiores, en el caso de GI el rango de variación es más amplio y la media presenta un importe superior.

\section{Gráfico 1. Distribución de la cuantía de la pensión según tipo (euros / año)}

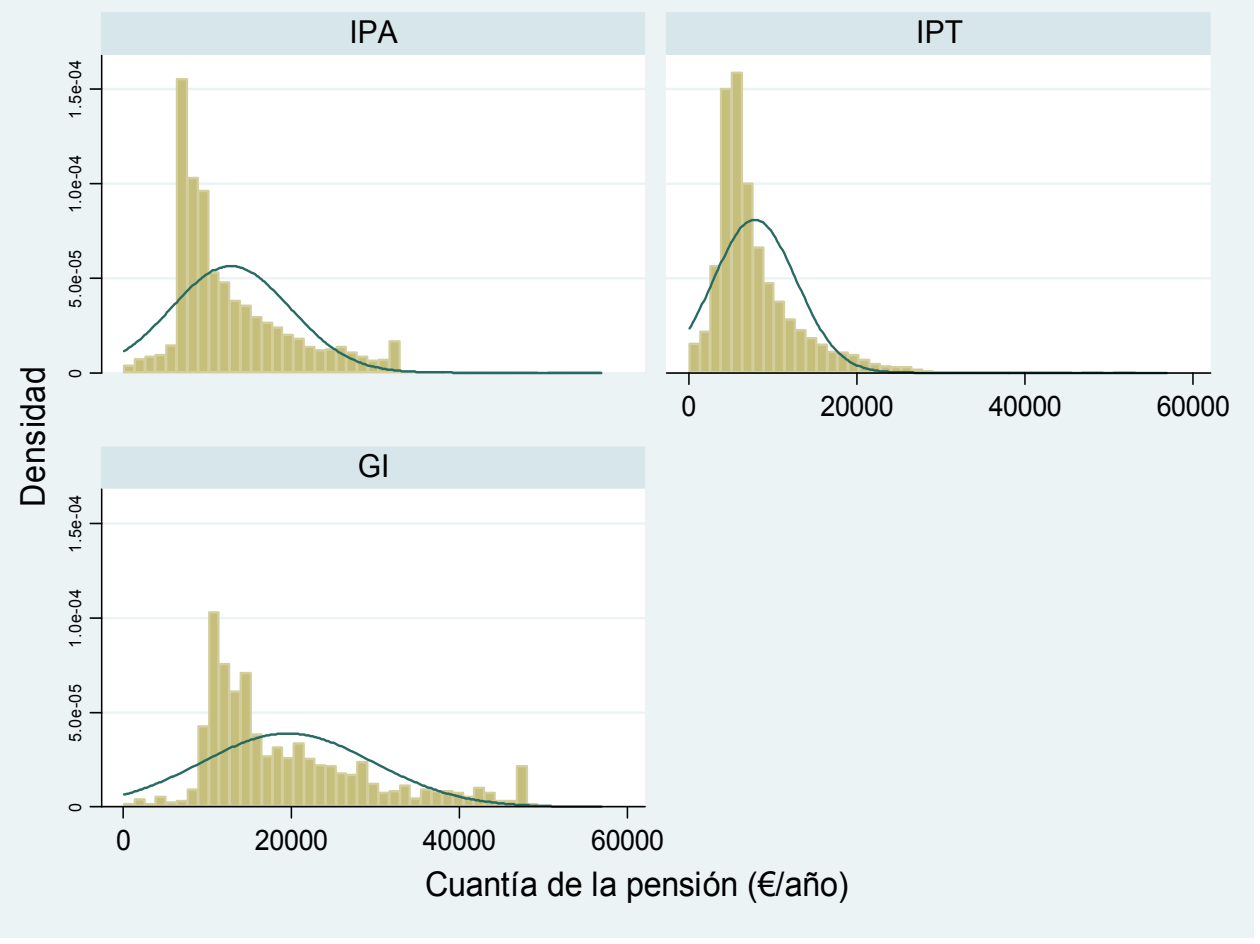

Dadas las cifras presentadas previamente relativas al porcentaje de pensionistas que tienen empleo, el incentivo a trabajar parece estar muy correlacionado con la cuantía de la pensión. La mayor proporción de trabajadores se observa para la IPT, que presenta respecto a la GI y la IPA un menor importe de la prestación (véase el Cuadro 3). No obstante, dado que la definición de incapacidad permanente se corresponde estrictamente con una "discapacidad para trabajar" estos resultados se podrían interpretar como una correlación de altos niveles de participación en el mercado de trabajo con menores niveles de discapacidad para trabajar. Además, el porcentaje de trabajadores entre las 
personas con GI (que tienen la mayor discapacidad y la mayor prestación) es ligeramente mayor que el grupo de IPA (como ya se vio en el Cuadro 1).

Si observamos la cuantía de la prestación según el grado y la situación de alta (últimas filas del cuadro 2), vemos que la pensión es mayor en el caso de las personas que no trabajan. La diferencia máxima se da en la GI y disminuye para la IPA y para la IPT, registrándose en este caso la menor brecha. Cuando el cálculo se realiza de forma separada para hombres y para mujeres, se observa que la diferencia es mayor para los hombres que para las mujeres, manteniéndose las distancias según el grado de la pensión. No obstante, debe recordarse que los que no trabajan han cotizado más años y son mayores, características ambas que inciden en el derecho a una pensión mayor.

En el Cuadro 3 se muestran las características de las personas objeto de análisis según el tipo de prestación que reciben. Se observa que las diferencias son escasas y se pueden resumir en un mayor número de hombres y un menor número de episodios de empleo cuanto mayor es la gravedad de la incapacidad permanente. Las restantes variables toman valores muy similares independientemente del tipo de pensión considerado. En general, las personas que no trabajan en 2006 han trabajado más años y son mayores, por lo que, en principio, podrían tener derecho a una pensión más elevada.

Si atendemos a las diferencias según el tipo de prestación y si la persona trabaja o no, los rasgos que diferencian a uno y otro grupo según la situación de alta son similares a los indicados para el total de pensionistas, independientemente del tipo de prestación. Así, el porcentaje de hombres es sustancialmente mayor entre las personas que trabajan (con la excepción de GI, si bien hay que tener en cuenta que el tamaño de este colectivo es pequeño). De la misma forma, los pensionistas que tienen empleo son personas más jóvenes, tanto en el momento actual como en el de reconocimiento de la IP.

En cuanto a su trayectoria laboral, el grupo de trabajadores ha empezado a trabajar a una edad ligeramente más temprana, han trabajado menos tiempo (tanto en términos absolutos como relativos), han tenido menos episodios de empleo y éstos han durado, en promedio, menos tiempo. Es decir, las diferencias clave están marcadas principalmente por la situación de alta en el empleo y no tanto por el tipo de prestación recibida.

Por último, cabe señalar que otro de los aspectos que puede contribuir a una diferente cuantía de la pensión y a un distinto incentivo a trabajar es el nivel educativo. La muestra de vidas laborales no proporciona datos sobre esta variable ${ }^{8}$. No obstante, se dispone de información sobre el grupo de cotización del trabajador, que puede interpretarse como una cierta aproximación del grado de cualificación del trabajador.

${ }^{8}$ La vinculación con el censo permite disponer del nivel educativo registrado en 1996 . No obstante el elevado número de casos perdidos y la falta de actualización del dato hace desaconsejable su uso. La propia documentación de la base de datos expresa serias dudas sobre la fiabilidad de esta variable. 
Para su análisis lo hemos reagrupado en cuatro categorías ${ }^{9}$. Se observa que para cualquier tipo de pensión, la cuantía de la misma es mayor en el grupo de alta y media cualificación, lo que parece indicar que el grupo de cotización es una variable que aproxima adecuadamente el nivel educativo o de cualificación de la persona.

Cuadro 3. Características de las personas que reciben prestación por incapacidad permanente según tipo de prestación y situación de alta en 2006

\begin{tabular}{|c|c|c|c|c|c|c|c|c|c|}
\hline & \multicolumn{3}{|c|}{ IPT } & \multicolumn{3}{|c|}{ IPA } & \multicolumn{3}{|c|}{ GI } \\
\hline & $\begin{array}{c}\text { No } \\
\text { trabaja }\end{array}$ & Trabaja & Total & $\begin{array}{c}\text { No } \\
\text { trabaja }\end{array}$ & Trabaja & Total & $\begin{array}{c}\text { No } \\
\text { trabaja }\end{array}$ & Trabaja & Total \\
\hline Sexo (\% hombres) & $64,2 \%$ & $88,2 \%$ & $68,0 \%$ & $69,6 \%$ & $78,6 \%$ & $69,7 \%$ & $77,6 \%$ & $76,5 \%$ & $77,6 \%$ \\
\hline \multicolumn{10}{|l|}{ Edad: } \\
\hline En 2006 & 54,2 & 47,4 & 53,1 & 53,5 & 48,4 & 53,4 & 51,7 & 46,1 & 51,6 \\
\hline En el 1er empleo & 25,1 & 20,3 & 24,4 & 24,0 & 21,5 & 24,0 & 23,6 & 22,5 & 23,6 \\
\hline En el $P^{o}$ de la IP & 47,7 & 38,1 & 46,2 & 45,7 & 36,4 & 45,6 & 42,6 & 32,4 & 42,4 \\
\hline $\begin{array}{l}\text { Discapacidad (en } \\
\text { algún empleo) }\end{array}$ & $0,8 \%$ & $1,5 \%$ & $0,9 \%$ & $1,6 \%$ & $4,4 \%$ & $1,7 \%$ & $2,3 \%$ & $0,0 \%$ & $2,3 \%$ \\
\hline \multicolumn{10}{|l|}{$\begin{array}{l}\text { Tiempo trabajado } \\
\text { (años) }\end{array}$} \\
\hline $\begin{array}{l}\text { Tiempo poten- } \\
\text { cial de trabajo } \\
\text { (hasta la IP) }\end{array}$ & 22,5 & 17,8 & 21,8 & 21,7 & 15,0 & 21,6 & 18,9 & 10,0 & 18,8 \\
\hline $\begin{array}{l}\text { Tiempo total } \\
\text { trabajado }\end{array}$ & 17,5 & 14,2 & 17,0 & 17,2 & 11,8 & 17,2 & 15,5 & 8,4 & 15,4 \\
\hline $\begin{array}{l}\text { Tiempo traba- } \\
\text { jado / tiempo } \\
\text { potencial }\end{array}$ & $77,6 \%$ & $79,9 \%$ & $81,8 \%$ & $79,3 \%$ & $79,1 \%$ & $77,9 \%$ & $81,8 \%$ & $84,1 \%$ & $79,3 \%$ \\
\hline$N^{0}$ de empleos & 11,5 & 11,0 & 11,4 & 10,1 & 8,3 & 10,1 & 8,6 & 3,6 & 8,5 \\
\hline $\begin{array}{l}\text { Duración media de } \\
\text { los empleos (años) }\end{array}$ & 4,3 & 2,9 & 4,1 & 4,5 & 3,2 & 4,5 & 4,2 & 3,4 & 4,2 \\
\hline $\begin{array}{l}\text { Recibe prestación } \\
\text { viudedad/orfandad }\end{array}$ & $3,3 \%$ & $1,2 \%$ & $3,0 \%$ & $2,4 \%$ & $1,3 \%$ & $2,4 \%$ & $1,8 \%$ & $17,6 \%$ & $2,0 \%$ \\
\hline $\begin{array}{l}\text { Cuantía de la } \\
\text { prestación }\end{array}$ & $7.994,07$ & $7.043,93$ & $7.854,07$ & $12.829,89$ & $10.291,50$ & $12.818,94$ & $19.891,05$ & $15.981,64$ & $19.889,54$ \\
\hline \multicolumn{10}{|c|}{ Cuantía de la prestación según grupo de cotización (aproximación del grado de cualificación del trabajador): } \\
\hline Alto & & & $14.344,64$ & & & $23.136,12$ & & & $31.985,49$ \\
\hline Medio & & & $9.539,82$ & & & $14.606,61$ & & & $22.758,08$ \\
\hline Bajo & & & $5.743,90$ & & & $9.016,38$ & & & $13.616,36$ \\
\hline Sin cotización & & & $7.210,08$ & & & $11.452,94$ & & & $18.834,95$ \\
\hline
\end{tabular}

Fuente: Cálculos de los autores a partir de la MCVL-2006.

${ }^{9}$ Véase el Cuadro A.1 en el Anexo. 


\subsection{Análisis econométrico}

En este epígrafe, estimaremos diferentes regresiones logísticas cuya variable dependiente es compatibilizar la pensión por incapacidad con el trabajo en 2006 frente a ser solamente pensionista por incapacidad ${ }^{10}$.

Las variables independientes son características personales (sexo, edad, discapacitado en algún momento antes de la prestación y si la persona vive sola en el hogar en 2006), características de la pensión (tipo, importe y si recibe otra pensión de viudedad $\mathrm{u}$ orfandad) y la trayectoria laboral hasta el reconocimiento de la incapacidad permanente (edad en el primer empleo y en el momento de reconocimiento de la IP, grupo de cotización en el último empleo, número de episodios de empleo y tiempo trabaja$\mathrm{do}^{11}$ ). Se realizan estimaciones diferentes según el tipo de pensión y según sexo.

En lugar de los coeficientes, se presentan los ratios de probabilidad relativa (odds ratios) para una interpretación más sencilla de los resultados ${ }^{12}$. Por ejemplo, puesto que el ratio de probabilidad relativa para la variable sexo es 3,7, la probabilidad de ser pensionista por incapacidad y trabajador es casi cuatro veces mayor para los hombres que para las mujeres. De la misma forma, vivir solo reduce un $16 \%(1 / 0,861=1,16)$ dicha probabilidad.

La IPT es el tipo de pensión con la mayor probabilidad relativa de estar trabajando, puesto que la multiplica por 17, respecto a la IPA, lo cual parece lógico dado que esta última debe recoger a personas con una discapacidad más grave. Con respecto a la cuantía de la prestación, para aquellos que tienen una pensión entre 3.600 y 14.400 euros al año la probabilidad de compatibilizar en 2006 es mayor respecto del resto. Aparentemente, podría estar detrás una lógica de "compensación": aquéllos con pensiones bajas completan respecto del nivel de ingresos deseado mediante un empleo. Sin embargo, este efecto "compensador" no existe para aquellos con pensión más baja (por debajo de 3.600 euros al año), que precisamente parece que serían los más proclives a simultanear pensión por incapacidad y empleo si esa explicación fuera la más acertada. Por tanto, la evidencia no muestra con total claridad que a mayor (menor) pensión por incapacidad existan menores (mayores) incentivos para compatibilizarla con un empleo. Volveremos sobre esta cuestión más adelante en las estimaciones separadas para cada tipo de pensión por incapacidad permanente.

El efecto de la edad en 2006 indica que los que tienen menos de 55 años tienen en torno al doble de probabilidad de simultanear pensión por incapacidad y empleo que

${ }^{10}$ Aunque se han dado cifras sobre el porcentaje de personas que compatibilizan empleo y pensión durante el tiempo trascurrido desde el reconocimiento de la misma, no se incluye en el análisis econométrico dado que los resultados pueden estar contaminados puesto que cuanto más tiempo hace que se tiene reconocida la IP, más probable es haber trabajado en algún momento.

${ }^{11}$ En el Cuadro A.1 del Anexo se muestran los estadísticos descriptivos de las variables.

${ }^{12}$ Los cuadros con los coeficientes y los errores estándar están a disposición de los interesados vía petición a los autores. Ocurre lo mismo con las estimaciones completas del Cuadro A.2. 
los que tienen entre 60 y 64 años, mientras que los que están entre 55 y 59 tienen una probabilidad prácticamente 1,7 veces mayor que los que están entre 60 y 64 .

Los resultados muestran que la probabilidad de compatibilizar pensión por incapacidad y trabajo disminuye con rapidez a medida que aumenta la edad en el momento en que se reconoce el derecho a la prestación. Es decir, el incentivo a trabajar se reduce con claridad cuando la persona está por encima de 55 años (la categoría de referencia).

Respecto a los efectos de la trayectoria laboral, la probabilidad de ser pensionista y trabajar en 2006 disminuye cuando las personas han entrado tarde al mercado de trabajo, cuando han tenido más episodios de empleo y han trabajado más tiempo. Por tanto, las personas con una menor vinculación al mercado laboral tienen una mayor probabilidad de compatibilizar ingresos provenientes del trabajo y de una prestación. Por otra parte, las personas que ya tenían una discapacidad con anterioridad a recibir la pensión es más probable que compatibilicen trabajo y pensión por incapacidad permanente. Nuestra interpretación es que aquellas personas que ya tenían una discapacidad antes del reconocimiento de la pensión desarrollarían estrategias personales que habrían llevado a saber introducirse y moverse en el mundo laboral (por ejemplo, en los procesos de selección de personal) y conseguir buscar de forma exitosa un empleo.

En cuanto al grupo de cotización (que usamos como aproximación del nivel de cualificación), las personas en el grupo alto o medio tienen mayor probabilidad de trabajar que aquéllas que están en el grupo de baja cualificación. Siguiendo la conocida teoría del capital humano, este resultado significaría que las personas con más alta cualificación normalmente tienen un menú de opciones de empleo más amplio y pueden acceder a puestos con salarios medios más elevados, lo cual incentivaría ceteris paribus la participación laboral frente a aquéllos individuos con baja o escasa cualificación. En definitiva, no trabajar tiene un coste de oportunidad más elevado para las personas con alta cualificación, lo cual las llevaría a compatibilizar pensión y empleo con una probabilidad mayor.

El modelo ha sido estimado de forma separada para hombres y para mujeres (cuadro 4) puesto que ambos géneros tienen historias y participaciones laborales diferentes. El signo de los coeficientes es similar para ambos, aunque hay diferencias relevantes en la magnitud de los ratios de probabilidad relativa.

En general, todos los efectos son mayores para los hombres que para las mujeres, especialmente en el caso de la cuantía de la pensión. Esta variable es estadísticamente significativa para los primeros pero no para las segundas. Como ocurre en el modelo estimado de forma conjunta, cuando la pensión es pequeña, la probabilidad de trabajar aumenta. No obstante, esta variable no afecta a la probabilidad de trabajar en el caso de las mujeres pensionistas.

Por otra parte, vivir solo (que puede considerarse una aproximación para el estado civil o para la existencia de otros ingresos en el hogar) contribuye positivamente a trabajar a la vez que se cobra la pensión en el caso de las mujeres mientras que el efecto es contrario en el caso de los hombres. 


\section{Cuadro 4. Determinantes de la compatibilidad entre pensión y trabajo según} sexo. Ratios de probabilidad relativa (odds ratios)

\begin{tabular}{|c|c|c|c|c|c|}
\hline & \multirow[t]{2}{*}{ Total } & \multicolumn{2}{|c|}{ Según sexo } & \multicolumn{2}{|c|}{ Según tipo de IP } \\
\hline & & Mujeres & Hombres & IPT & IPA \\
\hline Sexo (ref: mujer): & $3,718 * * *$ & & & $3,926 * * *$ & $1,850^{* * *}$ \\
\hline \multicolumn{6}{|c|}{ Tipo de pensión (Ref: IPA): } \\
\hline IPT & $16,971 * * *$ & $10,301 * * *$ & $19,125 * * *$ & & \\
\hline GI & $1,563 *$ & 2,421 & 1,393 & & \\
\hline \multicolumn{6}{|l|}{ Edad (Ref: 59-64): } \\
\hline$<45$ & $2,224 * * *$ & $2,300 * * *$ & $2,149 * * *$ & $2,349 * * *$ & 1,097 \\
\hline $45-49$ & $2,158 * * *$ & $2,762 * * *$ & $1.984 * * *$ & $2,262 * * *$ & 1,109 \\
\hline $50-54$ & $2,031 * * *$ & $2,136^{* *}$ & $1,958 * * *$ & $2,099 * * *$ & 1,295 \\
\hline $55-59$ & $1,676 * * *$ & 1,432 & $1,660^{* * *}$ & $1,691 * * *$ & 1,477 \\
\hline \multicolumn{6}{|c|}{ Edad en el 1er empleo(Ref: $>30)$ : } \\
\hline$<20$ & $1,625 * * *$ & 1,166 & $2,186^{* * *}$ & $1,703 * * *$ & 0,981 \\
\hline $20-24$ & $1,586 * * *$ & 1,323 & $2.018^{* * *}$ & $1,670^{* * *}$ & 1,006 \\
\hline $25-29$ & $1,264 *$ & 1,349 & $1,515^{* *}$ & $1,331 * *$ & 0,815 \\
\hline \multicolumn{6}{|c|}{ Edad en el $P^{0}$ de la IP (Ref: 56-64): } \\
\hline$<31$ & $18,618 * * *$ & $8,273 * * *$ & $22.251 * * *$ & $18,273^{* * *}$ & $46,820 * * *$ \\
\hline $31-45$ & $7,735 * * *$ & $3,703 * * *$ & $8,885^{* * *}$ & $7,473 * * *$ & $20,376^{* * *}$ \\
\hline $46-55$ & $3,054 * * *$ & $1,739^{*}$ & $3,397 * * *$ & $3,030^{* * *}$ & 4,996 \\
\hline \multicolumn{6}{|c|}{$N^{o}$ de episodios de empleo (Ref: $>20$ ): } \\
\hline$<5$ & $1,366 * * *$ & 1,189 & $1,473 * * *$ & $1,342 * * *$ & 1,819 \\
\hline $5-10$ & $1,381 * * *$ & 1,323 & $1,403 * * *$ & $1,382 * * *$ & 1,735 \\
\hline $11-20$ & $1,223 * * *$ & $1,751 * *$ & $1,162 * *$ & $1,188 * *$ & $2,262 * *$ \\
\hline \multicolumn{6}{|c|}{ Tiempo trabajado ( Ref: > 20 años): } \\
\hline Hasta 5 años & $0,691 * * *$ & 1,276 & $0,603^{* * *}$ & $0,636^{* * *}$ & 1,486 \\
\hline De 5 a 10 años & $0,794 * * *$ & 0,917 & $0,767 * * *$ & $0,777 * * *$ & 1,065 \\
\hline De 10 a 20 años & 0,912 & 0,862 & 0,922 & 0,901 & 1,147 \\
\hline \multicolumn{6}{|c|}{ Grupo de cotización (Ref: Baja): } \\
\hline Alta & $2,425 * * *$ & 1,298 & $2,699 * * *$ & $2,513 * * *$ & 0,820 \\
\hline Media & $1,516 * * *$ & $1,449 * * *$ & $1,542 * * *$ & $1,531 * * *$ & 1,221 \\
\hline Sin cotización & $1,327 * * *$ & 1,026 & $1,420^{* * *}$ & $1,365^{* * *}$ & 0,988 \\
\hline Cuantía de la pensió & & & & & \\
\hline
\end{tabular}




\begin{tabular}{|c|c|c|c|c|c|}
\hline$<3.600$ & $3,021 * *$ & 2,734 & $2,770 * *$ & 2,661 & 1,438 \\
\hline $3.600-7.200$ & $4,707 * * *$ & 2,660 & $5,131 * * *$ & $4,103^{* *}$ & 2,833 \\
\hline $7.200-14.400$ & $3,897 * * *$ & 2,369 & $4,110 * * *$ & $3,301^{*}$ & 3,167 \\
\hline $14.400-28.800$ & 1,533 & 1,659 & 1,526 & 1,332 & 1,569 \\
\hline Pensión de viudedad u orfandad & $1,406 *$ & 1,224 & 1,241 & 1,282 & 1,234 \\
\hline Vive solo & $0,858 * *$ & $1,885^{* * *}$ & $0,732 * * *$ & $0,869^{*}$ & 0,752 \\
\hline Discapacitado en algún empleo & $1,586 * *$ & 1,337 & $1,603^{* *}$ & $1,465^{*}$ & $3,045^{* * *}$ \\
\hline \multicolumn{6}{|l|}{ Comunidad Autónoma } \\
\hline Aragón & $2,121 * * *$ & $2,273 * *$ & $2,090 * * *$ & $2,238 * * *$ & 1,062 \\
\hline Asturias & $1,511 * * *$ & $2,385 * *$ & $1,435 * * *$ & $1,577 * * *$ & 0,815 \\
\hline Baleares & $1,806 * * *$ & 1,613 & $1,859^{* * *}$ & $1,791 * * *$ & 1,714 \\
\hline Canarias & 0,919 & 1,354 & 0,844 & 0,968 & 0,302 \\
\hline Cantabria & $1,735 * * *$ & 0,731 & $1,877 * * *$ & $1,670 * * *$ & $2,915^{*}$ \\
\hline Castilla La Mancha & $1,869 * * *$ & $2,068^{*}$ & $1,836^{* * *}$ & $1,927 * * *$ & 1,004 \\
\hline Castilla León & $1,668 * * *$ & $2,336^{* *}$ & $1,612^{* * *}$ & $1,680 * * *$ & 1,651 \\
\hline Cataluña & $1,771 * * *$ & $1,603 * *$ & $1,866^{* * * *}$ & $1,818 * * *$ & 1,312 \\
\hline Extremadura & $1,529 * * *$ & $2,205^{* *}$ & $1,463 * *$ & $1,561 * * *$ & 1,193 \\
\hline Galicia & 1,008 & 1,333 & 0,973 & 1,059 & 0,357 \\
\hline Madrid & $2,405 * * *$ & $2,4552^{* * *}$ & $2,430 * * *$ & $2,477 * * *$ & 1,748 \\
\hline Murcia & $2,189 * * *$ & $2,567 * * *$ & $2,115^{* * *}$ & $2,293 * * *$ & 1,445 \\
\hline Navarra & $2,216 * * *$ & 1,067 & $2,500 * * *$ & $2,261 * * *$ & 0,816 \\
\hline País Vasco & $1,865 * * *$ & $2,262 * * *$ & $1,819^{* * *}$ & $1,983 * * *$ & 0,386 \\
\hline La Rioja & $1,976 * * *$ & $3,646^{* *}$ & $1,840 * * *$ & $2,141 * * *$ & - \\
\hline Comunidad Valenciana & $1,649 * * *$ & $1,898 * * *$ & $1,586^{* * * *}$ & $1,606^{* * *}$ & $2,186^{* *}$ \\
\hline Ceuta y Melilla & 2,245 & 5,357 & 1,861 & $2,706^{*}$ & - \\
\hline \multicolumn{6}{|l|}{ Ref: Andalucía } \\
\hline $\mathrm{N}$ & 27.807 & 8.156 & 19.651 & 15.991 & 10.883 \\
\hline LR chi2(45) & $5.151,49$ & 503,38 & $4.271,68$ & $2.849,27$ & 184,58 \\
\hline Prob $>$ chi 2 & 0,000 & 0,000 & 0,000 & 0,000 & 0,000 \\
\hline Log likelihood & $-6.357,26$ & $-1.141,79$ & $-5.153,35$ & $-5.655,20$ & $-598,73$ \\
\hline Pseudo R2 & 0,288 & 0,180 & 0,293 & 0,201 & 0,134 \\
\hline
\end{tabular}

Fuente: Cálculos de los autores a partir de la MCVL-2006. 
La edad actual no afecta a la probabilidad de que las mujeres compatibilicen pensión y trabajo mientras que para los hombres esta probabilidad se reduce a medida que aumenta su edad. El efecto del resto de las variables es similar para hombres y mujeres en el sentido ya explicado para la muestra total.

Destaca la influencia que tiene la edad a la que se reconoce la IP, que multiplica casi por veinte la probabilidad de trabajar después de tener derecho a una IP de una persona joven respecto a los mayores de 55 años.

En resumen, las variables que afectan la probabilidad de que las mujeres compatibilicen pensión y trabajo se vinculan fundamentalmente a la edad y al tipo de pensión, mientras que en el caso de los hombres también influyen otras relacionadas con la trayectoria laboral y con la cuantía de la prestación.

También hemos realizado estimaciones separadas para cada tipo de pensión (dos últimas filas del cuadro 4). Conviene tener en cuenta que el tipo de prestación está muy relacionado (por definición) con la gravedad de la incapacidad y, en consecuencia, los efectos de las distintas variables podrían diferir en función del grado de gravedad de la discapacidad (que nosotros la observaríamos como diferentes tipos de pensión de incapacidad permanente). Así, la mayor parte de los que compatibilizan pensión por incapacidad permanente y empleo perciben pensiones por incapacidad permanente total, lo cual podría estar pesando en las estimaciones hasta el punto de que la compatibilización sólo fuera relevante para ese grupo y que los efectos estimados no se aplicaran en absoluto al resto de tipos de pensiones de incapacidad permanente. Adicionalmente, la cuantía de la prestación (si bien también depende de los años cotizados y de la base de cotización) se relaciona claramente con el tipo de pensión. En los modelos anteriores, la cuantía de la pensión era relevante para explicar la compatibilidad entre pensión y trabajo, indicando (aunque no de forma totalmente clara) que podría estar sucediendo una especie de compensación de ingresos, de manera que quienes buscarían un empleo serían aquellos que pretendieran complementar una pensión relativamente baja.

En estas estimaciones según tipo de incapacidad, sólo se presentan los casos de la IPA y la IPT, puesto que el número de personas es demasiado reducido para la GI. Se puede observar que mientras que para la IPT casi todas las variables incluidas tienen algún efecto sobre la compatibilización, no sucede así con la IPA; ahora bien cabe resaltar que se registran más diferencias en la significatividad estadística de los coeficientes que en su magnitud. Una de las variables clave es la edad en el reconocimiento de la pensión, de forma que las personas relativamente jóvenes en ese momento tienen mayores probabilidades de compatibilizar trabajo y prestación que las mayores (los menores de 31 años tienen 46, para IPA, ó 18 veces más probabilidad de trabajar que los mayores de 55 años, para IPT).

Ahora la cuantía de la prestación sólo influye sobre la compatibilización para el caso de la IPT, para los dos tramos intermedios de la pensión que ya vimos en las estimaciones para toda la población (lo cual refuerza la tesis que aquellos resultados estaban fuertemente determinados por el peso de los perceptores de IPT en la muestra total). A nuestro juicio, estos resultados pueden interpretarse como que el supuesto desincentivo a la participación en el mercado laboral que generaría una pensión más 
elevada no es tal y que la probabilidad de trabajar depende en primer lugar de la gravedad de la discapacidad sufrida, que lleva a cobrar un tipo de pensión u otra y, en segundo lugar, depende de la edad en el momento del reconocimiento de la incapacidad y de si se ha tenido alguna discapacidad en algún empleo anterior al reconocimiento de la discapacidad. Sólo para los que perciben la IPT (la que, por definición legal, está dirigida desde un principio a la posible compatibilización con el empleo) la cuantía de la pensión podría disminuir la probabilidad de participación por encima de 14.400 euros al año, pero también para los que cobran menos de 3.600 euros al año, lo cual forzosamente tiene que reflejar situaciones muy diferentes pues estos últimos no pueden estar haciendo un razonamiento relacionado con que no les merece la pena "completar" sus ingresos de la pensión con un empleo.

Por último, para comprobar si la cuantía de la pensión tiene un efecto desincentivador (para cada tipo de pensión de incapacidad permanente) entre los individuos más próximos a la edad de la pensión de jubilación, hemos realizado estimaciones adicionales para aquellos que se encuentran entre 50 y 64 años. Para el caso de la IPT los ratios reflejan sólo parcialmente ese supuesto efecto desincentivo aunque siempre con el intervalo más bajo de ingresos ofreciendo resultados contrarios a lo que se esperaría para un efecto desincentivo puro. No obstante, ninguno de los coeficientes de estas variables resulta ser estadísticamente significativo a los niveles habituales, por lo que no cabe hablar de influencia de la cuantía de la pensión sobre la compatibilización para los mayores de 50 años.

\subsection{La reforma de diciembre de 2007: una simulación}

En diciembre de 2007 se ha realizado una reforma de la Ley General de la Seguridad Social que modifica la regulación de la incapacidad permanente (Ley 40/2007, de 4 de diciembre, de medidas en materia de Seguridad Social). Los principales cambios son los siguientes: se establece un nuevo periodo de carencia para percibir prestaciones por IPT, IPA y GI derivadas de enfermedad común. De esta forma, si el individuo tiene menos de treinta y un años de edad será la tercera parte del tiempo transcurrido entre la fecha en que cumplió 16 años y la del hecho causante de la pensión. Si tiene cumplidos 31 años de edad será la cuarta parte del tiempo transcurrido entre la fecha en que se haya cumplido 20 y el día en que se hubiese producido el hecho causante, con un mínimo, en todo caso, de 5 años. Además, al menos la quinta parte del período de cotización exigible deberá estar comprendida dentro de los 10 años inmediatamente anteriores al hecho causante.

También se modifica el cálculo de la base reguladora para incapacidad permanente derivada de enfermedad común. Se calcula según lo establecido en el Cuadro 1 (véase el apartado 3) y al resultado obtenido se le aplica el porcentaje que corresponda en función de los años de cotización, según la escala prevista para la pensión de jubila- 
ción ${ }^{13}$, considerándose a tal efecto como cotizados los años que le falten al interesado, en el momento del hecho causante, para cumplir 65 años. En el caso de no alcanzarse 15 años de cotización, el porcentaje aplicable será del $50 \%$.

Por último, también establece que la cuantía de la pensión de incapacidad permanente total derivada de enfermedad común no podrá resultar inferior al $55 \%$ de la base mínima de cotización para mayores de 18 años, en términos anuales, vigente en cada momento. Además, si el trabajador es calificado de gran inválido, tiene derecho a una pensión vitalicia que se incrementa con un complemento, destinado a que el inválido pueda remunerar a la persona que le atienda. El importe de este complemento será equivalente al resultado de sumar el $45 \%$ de la base mínima de cotización vigente en el momento del hecho causante y el $30 \%$ de la última base de cotización del trabajador correspondiente a la contingencia de la que derive la situación de incapacidad permanente. En ningún caso el complemento señalado podrá tener un importe inferior al $45 \%$ de la pensión percibida, sin el complemento, por el trabajador. Esta norma elimina la posibilidad de cambiar el incremento de la pensión por el internamiento en un centro a cargo de la Seguridad Social ${ }^{14}$.

Con los datos de la Muestra Continua de Vidas Laborales, se verían afectados por la reforma un 35,6\% de las personas menores de 65 años que en 2006 percibían una pensión. Nótese que este colectivo lo estamos identificando en 2006, antes de la reforma de 2007, pero que cumple los requisitos de la reforma de $2007^{15}$.

Si bien esta reforma no está dirigida explícitamente a variar los incentivos a trabajar de los pensionistas por incapacidad, este resultado permitiría valorar la reforma realizada en términos de su eventual impacto sobre los incentivos a trabajar de los perceptores de pensiones por incapacidad permanente, porque se centra en un colectivo de personas que -antes de la implementación de la reforma- tiende a no compatibilizar la prestación con el empleo (tal como nos muestra el Cuadro A.4).

${ }^{13}$ Por los primeros 15 años cotizados: el $50 \%$. Por cada año adicional de cotización, comprendido entre el decimosexto y el vigésimo quinto, ambos incluidos: el $3 \%$. Por cada año adicional de cotización, a partir del vigésimo sexto: el $2 \%$, sin que el porcentaje aplicable a la base reguladora supere el $100 \%$.

${ }^{14}$ Para mayores detalles jurídicos de los cambios introducidos por la Ley 40/2007 véase, por ejemplo, Nicolás (2008).

${ }^{15}$ Es importante resaltar que no es posible realizar con los datos de 2008 de la Muestra Continua una evaluación del impacto de esta reforma. La propia Ley 40/2007 establece que para determinar la cuantía de las pensiones de IP derivada de enfermedad común que provengan de una IT iniciada con anterioridad a su entrada en vigor, serán de aplicación las normas anteriormente vigentes. Por tanto, hasta no disponer de la MCVL de 2010 no se podrá estudiar el efecto real de esta reforma (se debe tener en cuenta que para iniciar el procedimiento de reconocimiento de una IP deben haber transcurrido al menos 12 meses, con un máximo de 18, desde el inicio de la IT). Esos datos sólo podrán estar disponibles a finales de 2011 ó a principios de 2012. Por tanto, el uso de datos de 2006 es la mejor opción posible (o, al menos, la menos mala de las posibles). 
Para intentar aproximar los posibles efectos de esta reforma sobre la compatibilización de la pensión contributiva por incapacidad con un empleo se ha incluido una nueva variable ficticia en el modelo, que toma el valor 1 si la persona se ve afectada por el cambio y valor 0 en caso contrario. Los cambios, en términos de reducción de la prestación, afectan a las personas que han comenzado a trabajar después de los 30 años o a aquellas para las que, habiendo tenido su primer empleo antes de los 30 , la suma del tiempo trabajado, más los años que le faltan para cumplir 65 en el momento en que se reconoce la pensión, sea menor de 35. En todos los casos, el origen de la prestación se debe encontrar en una enfermedad común. Por otra parte, se establece una cuantía mínima para la pensión por IPT, que no podrá ser menor que el 55\% de la base mínima de cotización para trabajadores mayores de 18 años.

Hemos procedido a realizar una simulación del impacto del cambio legal de la siguiente forma: una vez identificados los trabajadores afectados, se ha calculado la cuantía que cobrarían si les fueran aplicados los cambios (en función del tiempo cotizado, la edad a la que empezaron a trabajar y el origen de la prestación). Con esta cuantía estimada, se ha estimado nuevamente una regresión logística (los resultados se muestran en el Cuadro A.5) a partir del cual se ha obtenido la probabilidad de compatibilizar pensión y empleo para determinados perfiles de trabajador, la cual se compara con la probabilidad derivada del modelo estimado con la cuantía real de la pensión en la sección previa.

Como se observa en el Gráfico 2 la probabilidad de compatibilizar empleo y pensión aumentaría tras la reforma, pasando de un $19,9 \%$ a un $28,3 \%$ para el grupo de referencia, es decir, aunque no sea el objeto de dicha reforma, sí contribuiría a fomentar la simultaneidad de ambas fuentes de ingresos de una manera sensible. Además, los cambios afectarían a determinados colectivos: principalmente las personas a las que se les reconoce el derecho a pensión antes de los 31 años, que incrementan en 11 puntos la probabilidad de compatibilizar pensión y trabajo. También se estima un incremento en dicha probabilidad en todos los tramos de edad a la que se empieza a trabajar, mayor cuanto menor es la edad de entrada al mercado laboral.

En cambio, las probabilidades según el tipo de pensión y el tramo de ingresos apenas sufren variaciones manteniéndose en las mismas cifras. Es decir, al igual que se veía en los modelos explicados previamente, el incentivo a compatibilizar pensión y empleo parece no derivarse de la cuantía de la prestación sino de otras variables, relacionadas principalmente con la edad a la que se entra en el mercado laboral o a la que se reconoce la pensión, las cuales han sido claves en la reforma realizada en 2007. Así pues, aunque no era su objetivo declarado, nuestra simulación predice un aumento de la compatibilización gracias al cambio legal de 2007, incidiendo así en un aumento de la integración laboral del colectivo de personas con discapacidad. 


\section{Gráfico 2. Probabilidad de compatibilizar pensión y empleo}

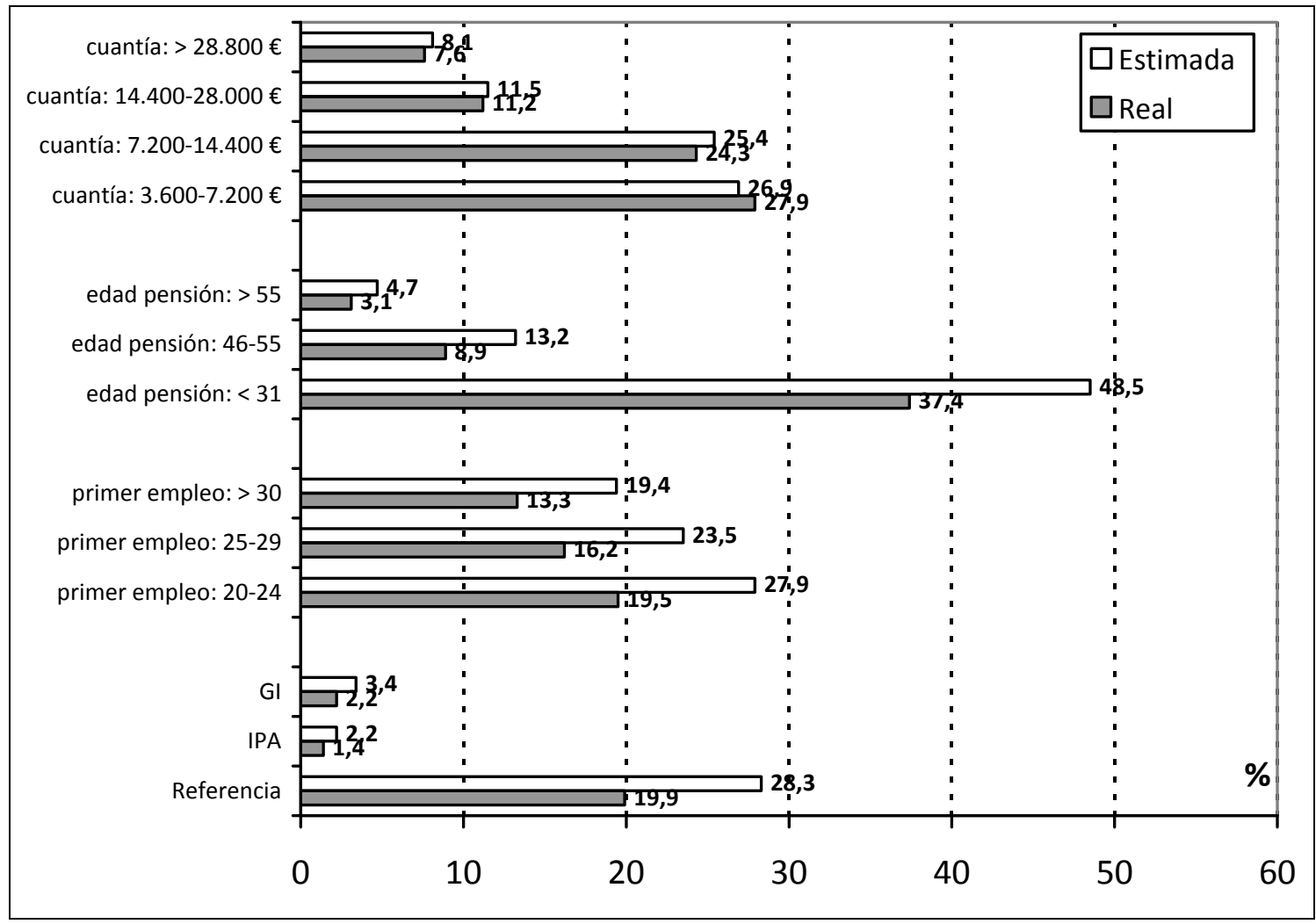

Referencia: hombre con IPT, de 45 a 55 años, que empezó a trabajar antes de los 20 años, se le reconoció la pensión en el tramo 31-45 años, cualificación media, con 5-10 episodios de empleo, 5-10 años trabajados, pensión menor de 3600 euros y en Andalucía.

\section{Conclusiones}

El objetivo con el que se ha planteado este artículo es estudiar la compatibilidad entre el trabajo remunerado y la percepción de prestaciones contributivas por incapacidad permanente. A partir de los datos de la Muestra Continua de Vidas Laborales se ha obtenido que un $9,9 \%$ de las personas menores de 65 que reciben una prestación por incapacidad permanente trabaja en 2006. Además, en el caso de la IPT, que por definición es la incapacidad más compatible con el trabajo, en 2006 estaban compatibilizando prestación y empleo un $15,6 \%$ de los beneficiarios. 
El análisis descriptivo muestra que en el grupo de pensionistas que trabajan hay más hombres y son relativamente más jóvenes. Asimismo, se han incorporado al mercado de trabajo y se les ha reconocido la IP a una edad más temprana. Sin embargo, a pesar de su pronta incorporación al empleo han trabajado menos tiempo y la duración media de sus episodios de empleo ha sido menor.

Aunque a nivel descriptivo se observa que las personas que no trabajan reciben pensiones de mayor cuantía, el análisis econométrico permite observar que un mayor importe de la pensión no tiene un efecto negativo general sobre la compatibilización, puesto que mientras que para importes anuales medio-bajos la probabilidad de compatibilizar es superior que para importes más elevados, en el caso de importes inferiores esta tendencia no se detecta. Es decir, una pensión elevada no desincentiva la participación en el mercado de trabajo, sino que la probabilidad de compatibilizar depende de la gravedad de la discapacidad sufrida, de la edad en el momento del reconocimiento de la pensión y de la experiencia anterior en la discapacidad. Este resultado es común a hombres y mujeres y para todos los tipos de IP. Ni siquiera para los trabajadores mayores de 50 años se ha encontrado una relación clara entre la cuantía de la prestación y la compatibilización.

Por otro lado, a medida que aumenta la edad a la que se reconoce la pensión disminuye la probabilidad de compatibilizar, mientras que las personas que se han incorporado más jóvenes pero que han trabajado menos tiempo tienen una mayor probabilidad de compatibilizar prestación y empleo, al igual que aquéllas que pertenecen al grupo de media o alta cualificación.

Finalmente, se ha realizado una simulación que intenta evaluar los posibles efectos que la reforma de las pensiones por incapacidad llevada a cabo en 2007 puede tener sobre la compatibilización entre pensiones y empleo. Las estimaciones permiten concluir que, aunque no sea su objetivo, esta reforma favorecerá con claridad la compatibilización entre prestación y empleo.

En definitiva, los resultados nos llevan a afirmar que si se quiere favorecer la compatibilización de prestación por incapacidad y empleo se debe incidir en aspectos de la carrera laboral, no en el importe de la prestación, el cual no parece tener un efecto desincentivador en la permanencia en el mercado de trabajo una vez reconocida una IP. Esta conclusión está en línea con lo ocurrido en otros mercados europeos donde se ha visto que rebajar las cuantías de las pensiones por incapacidad no conduce necesariamente a un aumento del empleo. De hecho, países como Luxemburgo que han eliminado las transferencias para las personas con capacidad parcial de trabajo (equivalente a la IPT en España) han experimentado caídas sensibles en las tasas de beneficiarios de prestaciones por discapacidad sólo para ver cómo esos descensos se trasladaban a aumentos en las tasas de beneficiarios de subsidios por desempleo (OCDE, 2007). Es el cambio de diseño de las pensiones (como hemos visto con la simulación de los efectos de los cambios legales de 2007 en España) lo que puede dar lugar a promocionar el empleo de las personas con discapacidad sin reducciones de las pensiones por incapacidad. No obstante, se necesita investigación adicional (sobre todo de carácter longitudinal gracias a nuevos datos) en esta misma línea para poder realizar análisis de los incentivos del diseño de estas pensiones para el empleo, no 
sólo en cuanto a su compatibilidad con las pensiones sino en cuanto a su eventual relación con la salida definitiva del mercado de trabajo y también en cuanto a si los resultados obtenidos se mantienen o son diferentes para las pensiones no contributivas.

\section{Bibliografía}

Álvarez de la Rosa, J.M. (1982). Invalidez permanente y Seguridad Social. Madrid: Civitas.

Blanco, A. (2000). The decision of early retirement in Spain. Documento de trabajo EEE76. FEDEA, Madrid.

Blanco Martín, J.M. (Coor.), (2002). Las incapacidades laborales. Un punto de vista práctico. Valladolid: Lex Nova.

Borovia Fernández, C. (Coor.), (2007). Valoración médica y jurídica de la incapacidad laboral. Madrid: La Ley.

Bound, J., Schoenmbaum, M., Stinebrickner. T.R. y Waidman, T. (1999). The dynamic effects of health on the labor force transitions of older workers. Labour Economics. Vol. 6, 179-202.

Bound, J. y Burkhauser, R.V. (1999). Economic Analysis of Transfer Programs Targeted on People with Disabilities. En O. Ashenfelter y D. Card, Handbook of Labor Economics, Vol. 3, (pp.3417-3528). Amsterdam: Elservier Science B.V.

Desdentado, A., Fernández, B. y Gonzalez-Sancho, E. (1986). La reforma de las pensiones de la Seguridad Social. Madrid: Civitas.

Díaz-Giménez, J. y Díaz-Saavedra, J. (2006). The Demographic and Educational Transitions and the Sustainability of the Spanish Public Pension System. Moneda y Crédito. Vol. 222, 223-270.

Jiménez, S. (2006). Evaluating the labor supply effects of alternative reforms of the Spanish pension system. Moneda y Crédito. Vol. 222, 271-312.

López Arriba, P.A. y Calvo Fernández, A. (1989). Evolución de la litigiosidad en España en materia de invalidez permanente (1977-1987). Revista de Trabajo, Núm. 93, 147-192.

Malo, M. A. (2004). ¿Cómo afectan las discapacidades a la probabilidad de ser activo en España? Un análisis empírico con datos de la Encuesta sobre Discapacidades. Deficiencias y Estado de Salud de 1999. Cuadernos de Economía. Vol. 27 Núm. 74, 75-108.

Nicolás Bernad, J.A. (2008). La incidencia de la reforma de la Seguridad Social por la Ley 40/2007. Información Laboral. Núm. 10, 2-47. 
OCDE (2007). New ways of addressing partial work capacity. París: OCDE. Disponible en la dirección electrónica http://www.oecd.org/dataoecd/6/6/38509814.pdf (último acceso: 23/04/2008).

Parsons, D. (1980). The Decline in Male Labor Force Participation. Journal of Political Economy. Vol. 88 Núm 1, 117-134. 


\section{Apéndice}

Cuadro A.1. Estadísticos descriptivos de la muestra

\begin{tabular}{|c|c|c|c|}
\hline & & Media & D.E. \\
\hline \multicolumn{2}{|l|}{ Trabaja en 2006} & 0,099 & 0,298 \\
\hline \multicolumn{2}{|l|}{ Trabajó desde la IP-2006 } & 0,222 & 0,415 \\
\hline \multirow{3}{*}{ Tipo de pensión: } & GI & 0,030 & 0,171 \\
\hline & IPA & 0,395 & 0,489 \\
\hline & IPT & 0,575 & 0,494 \\
\hline \multicolumn{2}{|l|}{ Sexo (\% hombres) } & 0,707 & 0,455 \\
\hline \multirow{5}{*}{ Edad } & $<45$ & 0,186 & 0,389 \\
\hline & $45-49$ & 0,132 & 0,339 \\
\hline & $50-54$ & 0,169 & 0,374 \\
\hline & $55-59$ & 0,233 & 0,423 \\
\hline & $59-64$ & 0,280 & 0,449 \\
\hline \multirow{4}{*}{$\begin{array}{l}\text { Edad en el 1er empleo (Edad calculada en el registro } \\
\text { del primer contrato) }\end{array}$} & $<20$ & 0,319 & 0,466 \\
\hline & $20-24$ & 0,320 & 0,466 \\
\hline & $25-29$ & 0,210 & 0,408 \\
\hline & $>30$ & 0,151 & 0,358 \\
\hline \multirow{4}{*}{$\begin{array}{l}\text { Edad en el Principio de la IP (Edad calculada en el } \\
\text { momento de reconocimiento de la IP) }\end{array}$} & $<31$ & 0,093 & 0,291 \\
\hline & $31-45$ & 0,384 & 0,486 \\
\hline & $46-55$ & 0,347 & 0,476 \\
\hline & $56-64$ & 0,175 & 0,380 \\
\hline \multirow{4}{*}{$\mathbf{N}^{\mathbf{o}}$ de empleos (Número de episodios de empleo) } & $<5$ & 0,325 & 0,468 \\
\hline & $5-10$ & 0,319 & 0,466 \\
\hline & $11-20$ & 0,205 & 0,404 \\
\hline & $>20$ & 0,151 & 0,358 \\
\hline \multirow{4}{*}{$\begin{array}{l}\text { Tiempo trabajado (Suma del tiempo trabajado en } \\
\text { todos los episodios de empleo anteriores a la IP, en } \\
\text { años) }\end{array}$} & Hasta 5 años & 0,092 & 0,288 \\
\hline & De 5 a 10 años & 0,190 & 0,392 \\
\hline & $\begin{array}{l}\text { De } 10 \text { a } 20 \\
\text { años }\end{array}$ & 0,361 & 0,480 \\
\hline & $>20$ años & 0,358 & 0,479 \\
\hline \multirow{4}{*}{$\begin{array}{l}\text { Grupo de cotización (Se corresponde con el grupo } \\
\text { del último contrato anterior al reconocimiento de la IP. } \\
\text { Recodificada en } 4 \text { niveles: } \\
\text { Alta cualificación (ingenieros y licenciados, ingenieros } \\
\text { técnicos y ayudantes y jefes administrativos); media } \\
\text { cualificación (ayudantes no titulados, oficiales admi- } \\
\text { nistrativos, subalternos, auxiliares administrativos, } \\
\text { oficiales de } 1^{\mathrm{a}} \text { y } 2^{\mathrm{a}} \text { y oficiales de } 3^{\mathrm{a}} \text { y especialistas); } \\
\text { baja cualificación (peones y asimilados, trabajadores } \\
\text { menores de } 18 \text { años y trabajadores por cuenta propia } \\
\text { en el REA) y sin cotización (determinados regímenes - } \\
\text { RETA y REEH- o situaciones - convenios especiales, } \\
\text { situaciones asimiladas al alta) }\end{array}$} & Alta & 0,052 & 0,223 \\
\hline & Media & 0,548 & 0,498 \\
\hline & Baja & 0,286 & 0,452 \\
\hline & Sin cotización & 0,114 & 0,318 \\
\hline \multirow{5}{*}{$\begin{array}{l}\text { Cuantía de la pensión (Importe anual total de la } \\
\text { prestación, en euros) }\end{array}$} & $<3.600$ & 0,050 & 0,218 \\
\hline & $3.600-7.200$ & 0,324 & 0,468 \\
\hline & $7.200-14.400$ & 0,401 & 0,490 \\
\hline & $14.400-28.800$ & 0,197 & 0,397 \\
\hline & $>28.800$ & 0,028 & 0,165 \\
\hline
\end{tabular}




\begin{tabular}{|c|c|c|c|}
\hline \multicolumn{2}{|c|}{$\begin{array}{l}\text { Pensión de viudedad u orfandad (En } 2006 \text { recibe prestaciones no } \\
\text { relacionadas con el trabajo) }\end{array}$} & 0,026 & 0,160 \\
\hline \multicolumn{2}{|c|}{ Vive solo (No convive con ninguna persona. Datos del padrón) } & 0,148 & 0,355 \\
\hline \multicolumn{2}{|c|}{$\begin{array}{l}\text { Discapacitado en algún empleo (En algún episodio de empleo anterior al } \\
\text { reconocimiento de la IP se registró una discapacidad superior al } 32 \% \text { ) }\end{array}$} & 0,015 & 0,120 \\
\hline \multirow{18}{*}{$\begin{array}{l}\text { Comunidad Autónoma } \\
\text { de la primera Afiliación }\end{array}$} & Andalucía & 0,219 & 0,413 \\
\hline & Aragón & 0,025 & 0,155 \\
\hline & Asturias & 0,040 & 0,197 \\
\hline & Baleares & 0,027 & 0,161 \\
\hline & Canarias & 0,034 & 0,182 \\
\hline & Cantabria & 0,016 & 0,125 \\
\hline & $\begin{array}{l}\text { Castilla La } \\
\text { Mancha }\end{array}$ & 0,033 & 0,178 \\
\hline & Castilla León & 0,051 & 0,220 \\
\hline & Cataluña & 0,179 & 0,384 \\
\hline & Extremadura & 0,025 & 0,156 \\
\hline & Galicia & 0,070 & 0,254 \\
\hline & Madrid & 0,088 & 0,284 \\
\hline & Murcia & 0,030 & 0,170 \\
\hline & Navarra & 0,013 & 0,112 \\
\hline & País Vasco & 0,054 & 0,227 \\
\hline & La Rioja & 0,007 & 0,081 \\
\hline & $\begin{array}{l}\text { Comunidad } \\
\text { Valenciana }\end{array}$ & 0,089 & 0,284 \\
\hline & Ceuta y Melilla & 0,002 & 0,042 \\
\hline $\mathbf{N}=\mathbf{2 7 . 8 0 7}$ & & & \\
\hline
\end{tabular}

Fuente: Cálculos de los autores a partir de la MCVL-2006. 
Cuadro A.2. Regresiones logísticas de la compatibilización entre pensión y empleo, por grupos de edad y tipo de pensión: Ratios de probabilidad relativa de la variable correspondiente a la cuantía de la pensión según edad y tipo de prestación

\begin{tabular}{|c|c|c|c|c|}
\hline & $>50$, IPA/GI & $>$ 50, IPT & $<50$, IPA/GI & $<50$, IPT \\
\hline & O.R. & O.R. & O.R. & O.R. \\
\hline \multicolumn{5}{|c|}{ Cuantía de la pensión (Ref: $>28.800$ ): } \\
\hline$<3.600$ & 0,655 & 1,495 & - & 0,447 \\
\hline $3.600-7.200$ & 0.643 & 1,949 & 1.605 & 1,367 \\
\hline $7.200-14.400$ & 1,117 & 1,473 & 1,740 & 1,373 \\
\hline $14.400-28.800$ & 1,225 & 0,788 & 1,573 & 1,125 \\
\hline
\end{tabular}

Nota: Ninguno de los coeficientes correspondientes en las estimaciones eran estadísticamente significativos a los niveles habituales. Las estimaciones completas están disponibles para los interesados.

Fuente: Cálculos de los autores a partir de la MCVL-2006.

Cuadro A.3. Efecto de la reforma de 2007: ratios de probabilidad relativa de la variable "afectado por la reforma"

\begin{tabular}{|c|c|c|}
\hline & & Trabaja en 2006 \\
\hline \multicolumn{2}{|l|}{ Total } & $0,678 * * *$ \\
\hline \multirow{2}{*}{ Según sexo } & Hombres & $0,636^{* * *}$ \\
\hline & Mujeres & 0,854 \\
\hline \multirow{3}{*}{ Según tipo de pensión } & IPT & $0,695 * * *$ \\
\hline & IPA & $0,364 * * *$ \\
\hline & GI & - \\
\hline
\end{tabular}

Nota: Las regresiones logísticas completas están a disposición de los interesados.

Fuente: Cálculos de los autores a partir de la MCVL-2006. 


\section{Cuadro A.4. Simulación del efecto de la reforma de 2007: Regresiones logísticas sobre la probabilidad de compatibilización de pensión y empleo}

\begin{tabular}{|c|c|c|c|}
\hline & Coef. & & S.E. \\
\hline \multicolumn{4}{|l|}{ Tipo de pensión (Ref: IPA): } \\
\hline IPT & $2,856 * *$ & $* * *$ & 0,097 \\
\hline GI & 0,441 & & 0,275 \\
\hline Sexo: hombre (ref: mujer) & $1,315 * *$ & $* * *$ & 0,067 \\
\hline \multicolumn{4}{|l|}{ Edad (Ref: 59-64): } \\
\hline$<45$ & $0,781 * *$ & $* * *$ & 0,116 \\
\hline $45-49$ & $0,760 * *$ & $* * *$ & 0,115 \\
\hline $50-54$ & $0,703 * *$ & $* * *$ & 0,108 \\
\hline $55-59$ & $0,515 * *$ & $* * *$ & 0,099 \\
\hline \multicolumn{4}{|c|}{ Edad en el primer empleo (Ref: $>30$ ): } \\
\hline$<20$ & $0,495 * *$ & $* * *$ & 0,137 \\
\hline $20-24$ & $0,471 * *$ & $* * *$ & 0,131 \\
\hline $25-29$ & $0,243 *$ & * & 0,133 \\
\hline \multicolumn{4}{|l|}{ Edad en el $\mathbf{P}^{\mathbf{0}}$ de la IP (Ref: 56-64): } \\
\hline$<31$ & $2,959 * *$ & $* * *$ & 0,179 \\
\hline $31-45$ & $2,090 * *$ & $* * *$ & 0,155 \\
\hline $46-55$ & $1,135 * *$ & $* * *$ & 0,144 \\
\hline \multicolumn{4}{|c|}{$\mathbf{N}^{0}$ de episodios de empleo (Ref: $>20$ ): } \\
\hline$<5$ & $0,320 * *$ & *** & 0,080 \\
\hline $5-10$ & $0,336 * *$ & $* * *$ & 0,073 \\
\hline $11-20$ & $0,212 * *$ & $* * *$ & 0,076 \\
\hline \multicolumn{4}{|l|}{ Tiempo trabajado (Ref: $>20$ años): } \\
\hline Hasta 5 años & $-0,373 *$ & $* * *$ & 0,108 \\
\hline De 5 a 10 años & $-0,213 *:$ & ** & 0,087 \\
\hline De 10 a 20 años & $-0,082$ & & 0,070 \\
\hline \multicolumn{4}{|l|}{ Grupo de cotización (Ref: Baja): } \\
\hline Alta & $0,862 * * *$ & $* * *$ & 0,141 \\
\hline Media & $0,401 * *$ & *** & 0,059 \\
\hline Sin cotización & $0,283 * *$ & *** & 0,079 \\
\hline \multicolumn{4}{|c|}{ Cuantía de la pensión (Ref: $>28.800)$ : } \\
\hline$<3.600$ & $1,497 * *$ & $* * *$ & 0,476 \\
\hline $3.600-7.200$ & $1,426 * *$ & $* * *$ & 0,434 \\
\hline $7.200-14.400$ & $1,345 * *$ & $* * *$ & 0,431 \\
\hline $14.400-28.800$ & 0,385 & & 0,436 \\
\hline Pensión de viudedad u orfandad & $0,339 *$ & * & 0,191 \\
\hline Vive solo & $-0,152 * *$ & ** & 0,068 \\
\hline Discapacitado en algún empleo & $0,449 *$ & ** & 0,181 \\
\hline \multicolumn{4}{|c|}{ Comunidad Autónoma (Ref: Andalucía) } \\
\hline Aragón & $0,736 * *$ & *** & 0,146 \\
\hline Asturias & $0,398 * *$ & $* * *$ & 0,126 \\
\hline Baleares & $0,592 * *$ & $* * *$ & 0,143 \\
\hline Canarias & $-0,092$ & & 0,148 \\
\hline Cantabria & $0,543 *$ & $* * *$ & 0,180 \\
\hline Castilla La Mancha & $0,627 * *$ & $* * *$ & 0,124 \\
\hline Castilla León & $0,497 * *$ & *** & 0,108 \\
\hline Cataluña & $0,564 * *$ & **** & 0,079 \\
\hline Extremadura & $0,428 * *$ & $* * *$ & 0,149 \\
\hline Galicia & 0,012 & & 0,108 \\
\hline Madrid & $0,866 *$ & **** & 0,092 \\
\hline Murcia & $0,792 * *$ & *** & 0,127 \\
\hline
\end{tabular}




\begin{tabular}{|l|r|r|}
\hline Navarra & $0,787^{* * *}$ & 0,195 \\
\hline País Vasco & $0,600^{* * *}$ & 0,111 \\
\hline La Rioja & $0,698^{* * *}$ & 0,242 \\
\hline Comunidad Valenciana & $0,491^{* * *}$ & 0,093 \\
\hline Ceuta y Melilla & 0,818 & 0,563 \\
\hline Constante & $-10,407$ & 0,479 \\
\hline & & \\
\hline N & 27.807 & \\
\hline LR chi2(46) & $5.142,26$ & \\
\hline Prob > chi2 & 0,0000 & \\
\hline Log likelihood & $-6.394,179$ & \\
\hline Pseudo R2 & 0,2868 & \\
\hline
\end{tabular}

Fuente: Cálculos de los autores a partir de la MCVL-2006. 